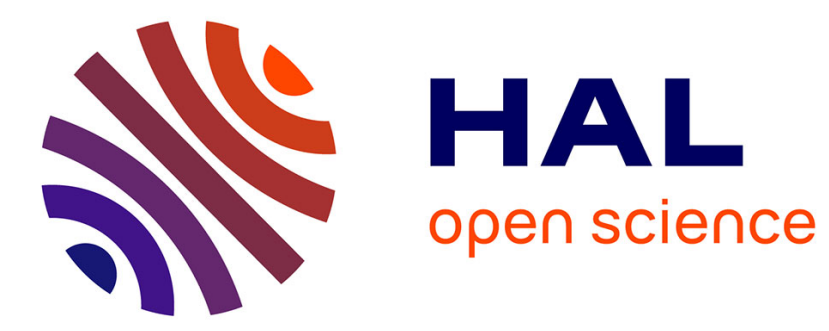

\title{
Dynamic Systems of Social Interactions
}

Ulrich Horst

\section{To cite this version:}

Ulrich Horst. Dynamic Systems of Social Interactions. Journal of Economic Behavior and Organization, 2010, 73 (2), pp.158. 10.1016/j.jebo.2009.09.007 . hal-00781340

\section{HAL Id: hal-00781340 \\ https://hal.science/hal-00781340}

Submitted on 26 Jan 2013

HAL is a multi-disciplinary open access archive for the deposit and dissemination of scientific research documents, whether they are published or not. The documents may come from teaching and research institutions in France or abroad, or from public or private research centers.
L'archive ouverte pluridisciplinaire HAL, est destinée au dépôt et à la diffusion de documents scientifiques de niveau recherche, publiés ou non, émanant des établissements d'enseignement et de recherche français ou étrangers, des laboratoires publics ou privés. 


\section{Accepted Manuscript}

Title: Dynamic Systems of Social Interactions

Authors: Ulrich Horst

PII:

DOI:

S0167-2681(09)00232-7

Reference: doi:10.1016/j.jebo.2009.09.007

JEBO 2453

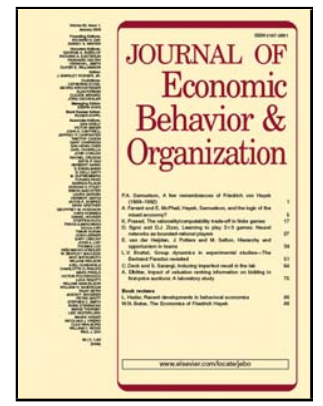

To appear in: Journal of Economic Behavior \& Organization

Received date: $\quad 14-6-2008$

Revised date: 23-9-2009

Accepted date: $\quad$ 23-9-2009

Please cite this article as: Horst, U., Dynamic Systems of Social Interactions, Journal of Economic Behavior and Organization (2008), doi:10.1016/j.jebo.2009.09.007

This is a PDF file of an unedited manuscript that has been accepted for publication. As a service to our customers we are providing this early version of the manuscript. The manuscript will undergo copyediting, typesetting, and review of the resulting proof before it is published in its final form. Please note that during the production process errors may be discovered which could affect the content, and all legal disclaimers that apply to the journal pertain. 


\title{
Dynamic Systems of Social Interactions
}

\author{
23rd September 2009
}

\begin{abstract}
We state conditions for existence and uniqueness of equilibria in evolutionary models with an infinity of locally and globally interacting agents. Agents face repeated discrete choice problems. Their utility depends on the actions of some designated neighbors and the average choice throughout the whole population. We show that the dynamics on the level of aggregate behavior can be described by a deterministic measure-valued integral equation. If some form of positive complementarities prevails we establish convergence and ergodicity results for aggregate activities. We apply our convergence results to study a class of population games with random matching.
\end{abstract}

JEL classification: C63, D50, D71.

Keywords: evolutionary dynamics, social interaction, equilibrium, interacting particle systems, coordination games. 


\section{Introduction}

A common observation in economics and the social sciences is the emergence of large differences in long run aggregate variables in the absence of corresponding differences in initial conditions $^{1}$. To accommodate this phenomenon, a model must generate an amplifier effect that transforms small changes in initial conditions into large changes in aggregate outcomes. Models of social interactions are capable of displaying amplifier effects. In these models an agent's behavior depends on the choices of other agents in some reference group and/or the distribution of actions throughout the whole population. In the presence of positive complementarities a change in initial conditions has a direct effect on the behavior of agents and an indirect effect through the interaction with others that are of the same sign. If these complementarities are powerful enough, initially small differences in individual behavior are amplified as time passes and largely different aggregate activities may emerge in the long run.

Much of the literature on social interactions assumes that interactions are either local or global. Agents interact locally when each agent interacts with a small stable set of peers in an otherwise large economy. Local interactions are designed to capture economic environments where markets do not exist to mediate all of the agents' choices. Agents interact globally if they only care about the distribution of actions or preferences throughout the population. Global interactions naturally capture market interactions and uniform random matching.

Models of purely local and purely global interactions are well understood; see, for instance, Kandori et al. (1993), Blume (1993), Benaim and Weibull (2003) and references therein. In many applications it is natural, though, to combine local and global interactions in order to integrate non-market interactions with market interactions or neighborhood effects with global externalities. Local technological complementarities, for instance, have been identified as a possible determinant of economic growth (Durlauf 1993), but firms also compete in the world markets; rumors may be transmitted both locally by word-of-mouth communication (Kosfeld 2005) and globally when agents meet at random (Banerjee 1993); counterparty relations between individual business partners are important channels for the contagious spread of financial distress across firms (Allen and Gale 2000), but financial distress may also spread through the impact of a company's financial health on the overall business climate (Horst 2007). Financial market models also call for a combination of local and global interactions. Although asset prices clearly depend on the average expectation of future prices and dividends throughout the entire set of market participants, there is also a

\footnotetext{
${ }^{1}$ Important examples which have been studied using microeconomic interaction models include the substantial difference in output levels and growth rates of neighboring countries (Durlauf 1993), the gradual emergence of "good" and "bad" neighborhoods in initially homogeneous cities (Topa 2001) and contagious deteriorations of credit ratings resulting from small economic shocks (Horst 2007).
} 
large literature on agent-based models of financial markets which has shown that contagious interaction and imitation effects between individual traders may cause asset prices to deviate from their fundamental values and cause bubbles and crashes.

Models of local and global interactions have only recently been studied in the economics literature. Horst and Scheinkman $(2006,2007)$ proved existence and convergence results for equilibria in static economies with an infinity of locally and globally interacting agents. This paper considers a dynamic discrete choice version of their model. Following Blume (1993), we assume that agents face repeated binary choice problems. They change their actions at random points in time at a rate that depends on the current states of some neighbors and the average situation throughout the entire population. The randomness in the agents' choices prevents their actions from converging pathwise to some steady state, so an appropriate notion of equilibrium is not a particular state but rather an invariant distribution for the microscopic process of individual states.

There is a large literature (Foster and Young 1990, Kirman 1992, Young 1993) on stochastic stability in evolutionary models that uses stationary distribution to describe the long run behavior in economic models. Existence and uniqueness results for stationary distributions typically require some form of continuity of the agents' transition rates, i.e., that an individual's behavior depends only weakly on the choices of far away agents. Due to the macroscopic dependence of the agents' behavior on aggregate quantities, such a condition is not satisfied in our model. To overcome this problem we apply the method of separating the local and global interaction of Föllmer and Horst (2001). The idea is to view the microscopic process of all the individual agents' states as a process of purely local interaction with "global externalities" where the externalities describe the evolution of aggregate behavior. Such a separation of the local and global interaction component is not necessary in models with a finite population (Ioannides and Soetevent 2005, Ioannides 2006). However, when global interactions are present the analysis is more naturally done in the context of an infinity of agents where a law of large numbers applies ${ }^{2}$. In fact, we prove that despite the strong correlations between individual choices, aggregate quantities follow a deterministic dynamics. This implies that our model can be viewed as a time-inhomogeneous model of local interaction where the inhomogeneity describes the evolution of the aggregate behavior.

If some form of positive complementarities prevails and the flip rates are strictly positive, we establish the existence of maximal and minimal equilibria. Under a weak interaction condition the equilibrium is unique. In this case aggregate quantities settle down to a unique limit in the long run. Uniqueness breaks down if the interaction becomes too strong. These results are applied to a class of local interaction games with random matching. Specifically,

\footnotetext{
${ }^{2}$ For economic models with an infinity of locally and globally interaction agents the separation approach has previously been applied by Horst (2005) and Bisin et al. (2006)
} 
we introduce an "anonymous" component into the model of Blume $(1993,1995)$ and study its impact on the long run behavior of aggregate play. It turns out that our model displays a much richer dynamics than Blume's models of purely local interactions. We prove that aggregates settle down to a unique limit in the long run when the interactions are sufficiently weak and that and illustrate that two stable equilibria coexist when the intensity of choice increases. Our numerical simulations also illustrate that self-reinforcing global feedback effects may prevent the agents from coordinating on the risk dominant equilibrium when the impact of the global interaction component is too powerful. In this case small differences in initial conditions are amplified as time passes and very different dynamics emerge in the long run. Such a dependence of the long run dynamics on initial conditions is a typical feature of meanfield models; this feature is not observed in Blume's local interaction model. In particular, in our framework the selection of equilibrium result is not always robust to local vs. global interaction. This distinguishes our results from those of Ellison (2000).

The remainder of this note is organized as follows. Dynamic systems of social interactions are introduced in Section 2. Section 3 states existence and uniqueness results of equilibrium for monotone systems. Section 4 outlines an application to population games.

\section{Dynamic Systems with Discrete Choice}

In this section we define dynamic systems of social interactions. The agents are located on the d-dimensional lattice with integer entries $\mathbb{A}$ and choose actions from the binary set $C=\{-1,+1\}$. A configuration is a list of actions for each agent. The configuration space

$$
E:=\left\{\eta=\left(\eta^{a}\right)_{a \in \mathbb{A}}: \eta^{a} \in C\right\}
$$

is equipped with the product topology and hence it is compact. The agent $a \in \mathbb{A}$ myopically switches her state at Poisson random times at a rate $c(\eta, a)$.

Remark 2.1 If the rates of switching from +1 to -1 and from -1 to +1 sum up to one this is equivalent to saying that choice opportunities for the agent arise at Poisson random times with rate one and when such an opportunity arises she switches her state with probability $c(\eta, a)$.

The agent's transition rate depends on the configuration $\eta$ both locally through the actions taken by the agents in her neighborhood

$$
N(a):=\{b:|a-b|=1\}
$$

and globally through the distribution of choices throughout the entire population. To accommodate the global component of the interaction we assume that the flip rates depend on 
the empirical field $R(\eta)$ associated with an action profile $\eta \in E$, i.e.,

$$
c(\eta, a) \equiv c(\eta, a, R(\eta)) .
$$

The empirical field $R(\eta)$ is a probability measure on $E$. It is formally defined in (9); at this point it should only be viewed as an object that carries all macroscopic information about $\eta$.

\subsection{Separating local and global interactions}

The general form of the flip rates (2) is not yet convenient for proving the existence of equilibrium distributions for the microscopic process $\left\{\eta_{t}\right\}$ that describes the evolution of all the individual sates. If the flip rates depend on aggregate quantities in a non-trivial manner they are not continuous in the product topology so standard existence and uniqueness results for stationary distributions of the Markov processes $\left\{\eta_{t}\right\}$ do not apply; even the definition of the process is delicate. To overcome these difficulties we define the flip rates as continuous mappings $c(\cdot, a, \cdot): E \times \mathcal{M}_{h} \rightarrow \mathbb{R}_{+}$on the extended state space $E \times \mathcal{M}_{h}$. Here $\mathcal{M}_{h}$ denotes the class of all spatially homogeneous probability measures on $E$ equipped with the topology of weak convergence. The flip rates $c(\eta, a, \mu)$ describe the transition of choices in a benchmark model of purely local interactions when the agents' assessment about the overall distribution of choices is "frozen" to $\mu$ and does not change over time. They satisfy the Markov property

$$
c(\eta, a, \mu)=c(\xi, a, \mu) \quad \text { if } \quad \eta^{b}=\xi^{b} \text { for } b \in N(a)
$$

In order to construct a model where at any point in time the instantaneous flip rate is of the form (3) with $\mu$ replaced by the prevailing empirical field we need to impose some form of spatial homogeneity. Otherwise there is no reason to assume that configurations have empirical averages. We therefore assume that all agents are identical ex-ante so the flip rates are invariant with respect to simultaneous shifts of agents and configurations. ${ }^{3}$

Definition 2.2 A dynamic system of social interactions is defined by a family of translation invariant flip rates $\{c(\eta, a, \mu)\}_{\eta \in E, a \in \mathbb{A}, \mu \in \mathcal{M}_{h}}$ that satisfy (3).

We denote the generator associated with the rates $\{c(\eta, a, \mu)\}_{\eta \in E, a \in \mathbb{A}}$ by $A_{\mu}$. It induces a unique time-homogeneous Markov process on the configuration space. If the agents' assessments about aggregate behavior follow some exogenous measure-valued process $\left\{\Psi_{t}\right\}$, then

\footnotetext{
${ }^{3}$ This assumption will guarantee that the distribution of choices is homogeneous so that spatial averages exist. If the distribution is not homogeneous, averages may not exist. Consider, for instance, the measure $\nu$ that puts all mass on the configuration $\eta$ defined by $\eta^{a}=+1$ if $a \in B_{2 n+1} \backslash B_{2 n}$ for some $n \in \mathbb{N}$ and $\eta^{a}=-1$

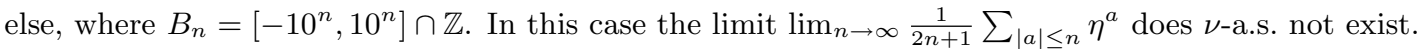


the family of time dependent operators $\left\{A_{\Psi_{t}}\right\}$ generates a locally interacting Markov process $\left\{\eta_{t}\right\}$ with time-inhomogeneous transition dynamics:

$$
\mathbb{P}\left[\eta_{t+h}^{a} \neq \eta_{t}^{a} \mid \eta_{t}\right]=c\left(\eta_{t}, a, \Psi_{t}\right) h+o(h) \quad \text { as } \quad h \downarrow 0 .
$$

While the construction of the process with purely local interactions is standard the construction in the presence of global interactions requires an additional continuity condition on the dependence of the flip rates on the macroscopic component. To this end, let

$$
\Delta_{y}(f):=\sup \left\{|f(\eta)-f(\xi)|: \eta^{x}=\xi^{x} \forall x \neq y\right\} \quad \text { and } \quad \mu(f):=\int f d \mu
$$

denote the oscillation at site $y$ of the function $f$ on $E$ and the integral of $f$ with respect to $\mu$, respectively. We fix some constant $r>0$ and recall from Horst (2002) that the metric

$$
d_{r}(\mu, \nu):=\sup _{f \in \mathcal{L}} \frac{|\mu(f)-\nu(f)|}{\sum_{y} 2^{r|y|} \Delta_{y}(f)}
$$

induces the weak topology on $\mathcal{M}_{h}$. We are now ready to state our continuity condition.

Assumption 2.3 The flip rates depend in a Lipschitz continuous manner on $\mu$, i.e.,

$$
|c(\cdot, \cdot, \mu)-c(\cdot, \cdot, \nu)| \leq L d_{r}(\mu, \nu) .
$$

The preceding assumption is satisfied if, for example, the flip rates depend on $\mu$ only through the expected behavior

$$
m(\mu):=\int \eta^{0} \mu(d \eta)
$$

of an individual agent under $\mu$ and if this dependence is Lipschitz continuous. We illustrate this property by means of the following example.

Example 2.4 Suppose that the agents are located on the two-dimensional integer lattice, that $c(\eta, a, \mu)$ depends on $\mu$ only through $m(\mu)$ and that the flip rates take the form of an Ising model of statistical mechanics as in Blume (1993) or Föllmer (1974):

$$
c(\eta, a, \mu)=\frac{1}{1+\exp \left[2 \beta \eta^{a}\left(h+J_{1} m(\mu)+J_{2} \sum_{b \in N(a)} \eta^{b}\right)\right]} \quad(\beta>0) .
$$

Here $J_{2} \geq 0$ measures an agent's desire for conformity. The quantity $h+J_{1} m(\mu)$ can be viewed as an intrinsic value associated with her choice. It depends on the distribution of choices in the population. The relative importance of the global component is measured by the non-negative parameter $J_{1}$. The case $J_{1}=0$ corresponds to model of purely local interactions while $J_{2}=0$ yields a continuous time version of the mean-field model of Brock and Durlauf (2001). Since 
the function on the right hand side of equation (7) is uniformly (in $\eta$ ) Lipschitz continuous in $m(\mu)$ with some constant $L$ and the projection mapping $f(\eta)=\eta^{0}$ satisfies $\Delta_{0}(f)=2$ and $\Delta_{x}(f)=0$ for $x \neq 0$, we see that

$$
|c(\eta, a, \mu)-c(\eta, a, \nu)| \leq L\left|\int f d \mu-\int f d \nu\right| \leq 2 L d_{r}(\mu, \nu) .
$$

\subsection{The microscopic and the macroscopic process}

Before proceeding with our analysis of the population dynamics, let us formally introduce the notion of an empirical field. To this end, let $\mathcal{M}_{0}$ be the class of all ergodic probabilities on $E$. A probability measure is called ergodic, if it is trivial on the $\sigma$-field of all shift invariant events. The ergodic theorem states that if $\mu \in \mathcal{M}_{0}$, then spatial averages exist $\mu$-a.s. and equal their expected values under $\mu$. Furthermore, ergodic measures are concentrated on the set $E_{0}$ of all ergodic configurations whose associated empirical field defined as the weak limit

$$
R(\eta):=\lim _{n \rightarrow \infty} \frac{1}{\left|\mathbb{A}_{n}\right|} \sum_{a \in \mathbb{A}_{n}} \delta_{\tau^{a} \eta}(\cdot)
$$

exists along the increasing sequence $\mathbb{A}_{n}:=[-n, n]^{d} \cap \mathbb{A}$ and is ergodic. Here $\delta_{\eta}(\cdot)$ denotes the Dirac measure that puts all mass on $\eta$, and $\tau^{a}$ is the canonical $a$-fold shift operator defined by $\tau^{a} \eta=\left(\eta^{a+b}\right)_{b \in \mathbb{A}}$. The empirical field carries all macroscopic information about $\eta$.

Example 2.5 The average action associated with an ergodic configuration $\eta \in E_{0}$ is given by the integral of the projection mapping $f(\xi)=\xi^{0}$ with respect to $R(\eta)$, i.e.,

$$
\int f d R(\eta)=\lim _{n \rightarrow \infty} \frac{1}{\left|\mathbb{A}_{n}\right|} \sum_{a \in \mathbb{A}} \eta^{a} .
$$

We are now ready to state the main results of this section. Outlines of the proofs are given the appendix.

Theorem 2.6 Let the flip rates $c(\eta, x, \mu)$ satisfy Assumption 2.3 and the initial distribution $R$ be ergodic. There exists a unique Markov process $\left\{\eta_{t}\right\}$ on the state space $E_{0}$ such that

$$
\mathbb{P}\left[\eta_{t+h}^{a} \neq \eta_{t}^{a} \mid \eta_{t}=\eta\right]=c(\eta, a, R(\eta)) h+o(h) \quad \text { as } \quad h \downarrow 0 .
$$

The preceding theorem states that under Assumption 2.3 the microscopic processes $\left\{\eta_{t}\right\}$ exists and induces the macroscopic process $\left\{R\left(\eta_{t}\right)\right\}$ of ergodic empirical fields. Ergodicity of the macroscopic process implies that the configurations have spatial averages at any point in time. The next result shows that aggregates evolve through time as if they were deterministic. Moreover, empirical fields are a sufficient statistic: knowledge of the current empirical field 
is enough to predict aggregates at future times; such a recursion typically does not hold on the level of empirical averages. ${ }^{4}$ Our model can hence be viewed as a model of purely local interactions with a time-inhomogeneous externality generated by the global interaction.

Theorem 2.7 Under the assumption of Theorem 2.6 the macroscopic process $\left\{R\left(\eta_{t}\right)\right\}$ is given as the unique solution to the measured-valued integral equation

$$
R_{t}(f)=R(f)+\int_{0}^{t} R_{u}\left(A_{R_{u}} f\right) d u \quad(f \in \mathcal{C}) .
$$

For a model of mean-field interactions where the agents only care about the distribution of choices in the population the integral equation in (10) reduces to an ordinary differential equation. In this case the microscopic process for an individual agent can also be constructed by analogy to the so-called Mckean process or, more directly, using Kurtz's (1978) strong approximation result for Poisson processes by Browninan motion. The former method has recently been applied by Tanabe (2006) to prove propagation of chaos results of large economies. Both approaches are based on an approximation of infinite economies by large but finite ones. They apply only if the individual states are asymptotically independent. This assumption is satisfied for mean field models. It is not satisfied for models of local interactions where the states of neighboring agents are correlated. For mean-field models all three approaches (Mckean process, strong approximation, and empirical fields) are equivalent.

Remark 2.8 Ordinary differential equations commonly arise as limiting objects in discretetime models of adaptive learning and fictitious play. In both cases the state is a vector of accumulated empirical frequencies of strategies used in the previous rounds. Our approach is qualitatively quite different. The macroscopic process describes the evolution of spatial (population) rather than temporal averages. As such it is conceptually much closer to that of Benaim and Weibull (2003). These authors consider a sequence of discrete time mean-field models and study the evolution of population averages. Their ODE arises in the limit of an infinite population after suitable scaling.

\section{Equilibria of Monotone Systems}

In a random economy with many agents where probabilistic choices prevent configurations from converging pathwise to some steady state, an appropriate notion of equilibrium is not a particular state, but rather a distribution of states which reflects the proportion of time

\footnotetext{
${ }^{4}$ Loosely speaking, to forecast the distribution of states tomorrow one needs to know what proportions of agents have neighbors configured in the various states today. This information is not carried in the empirical distribution even if the flip rates depend only on average actions.
} 
the agents spend in the states. This calls for an existence result for invariant distributions for the microscopic process. When started with an invariant measure $\mu$ the distribution of individual choices does not change over time. Since any invariant measure can be written as a convex combination of ergodic ones we may with no loss of generality assume that $\mu$ is ergodic. In this case the empirical fields satisfy $R\left(\eta_{t}\right) \equiv \mu$ so aggregate quantities are in a steady state while individual choices fluctuate in accordance with $\mu$.

Definition 3.1 A probability measure $\mu^{*}$ on $E$ is an equilibrium of the system $\{c(\eta, x, \mu)\}$ if $\mu^{*}$ is an ergodic stationary distribution for the associated microscopic process. It is globally stable if the macroscopic process converges to $\mu^{*}$ independently of the initial conditions.

We assume that an agent is more likely to flip to the opposite state in a short period of time if she generally disagrees with her environment than if she generally agrees with it. In a benchmark model of purely local interactions this translates into the following condition on the flip rates: if $\eta^{a} \leq \xi^{a}$ for all $a \in N(x)$, then

$$
c(\eta, x, \mu) \leq c(\xi, x, \mu) \text { if } \eta^{x}=\xi^{x}=-1 \quad \text { and } \quad c(\eta, x, \mu) \geq c(\xi, x, \mu) \text { if } \eta^{x}=\xi^{x}=1 .
$$

In order to state a corresponding condition when the agents also react to the population average, we write $\nu \geq \mu$ for two probability measures if the integrals of monotone functions with respect to $\nu$ dominate the integrals of the same functions with respect to $\mu$. The idea that an agent's propensity to switch to a different state increases in the number of neighbors and the proportion of agents configured in that state is captured by the following definition.

Definition 3.2 The system is monotone if $\nu \geq \mu$ and $\eta^{a} \leq \xi^{a}$ for all $a \in N(x)$ implies

$$
c(\eta, x, \mu) \leq c(\xi, x, \nu) \text { if } \eta^{x}=\xi^{x}=-1 \quad \text { and } c(\eta, x, \mu) \geq c(\xi, x, \nu) \text { if } \eta^{x}=\xi^{x}=1 .
$$

Our monotonicity condition can be viewed as a version of the positive complementarity condition commonly assumed in the theory of supermodular games, ${ }^{5}$ suitably adapted to capture the random and discrete nature of the agents' choice dynamics. In supermodular games an agent's utility of increasing an action is increasing in the other players actions while in our model an agent's propensity to switch to a higher state is increasing in the number of agents configured in the "plus" state. If the flip rates were differentiable, assumption (12) would translate into a positivity condition on the cross-partial derivatives of the rate function with respect to an agent's own state and, respectively, the neighbors' choices and the average.

The monotonicity condition allows us to prove that when started in an "all low" or "all high" configuration, aggregate quantities settle down in the long run.

\footnotetext{
${ }^{5}$ See, e.g., Milgrom and Roberts (1990), Topkis (1979) and Vives (1990) and references therein.
} 
Theorem 3.3 Let $\left\{R_{t}^{ \pm \mathbf{1}}\right\}$ be the macroscopic processes of a monotone system when $\eta_{0}^{a} \equiv \pm 1$.

(i) For all $s \leq t$ the processes $\left\{R_{t}^{-\mathbf{1}}\right\}$ and $\left\{R_{t}^{+\mathbf{1}}\right\}$ satisfy the monotonicity condition

$$
R_{s}^{-1}(f) \leq R_{t}^{-1}(f) \quad \text { and } \quad R_{s}^{1}(f) \geq R_{t}^{+1}(f)
$$

for every continuous function $f$ on $E$ for with $f(\eta) \leq f(\xi)$ if $\eta^{a} \leq \xi^{a}$ for all $a \in \mathbb{A}$.

(ii) The weak limits $\underline{\mu}:=\lim _{t \rightarrow \infty} R_{t}^{-1}$ and $\bar{\mu}:=\lim _{t \rightarrow \infty} R_{t}^{+1}$ exist.

The link between supermodular games and our dynamic discrete choice model suggests that the existence result for smallest and largest equilibria in supermodular games carries over to our framework. This is in fact true provided the weak limits $\mu$ and $\bar{\mu}$ are ergodic measures. In this case the set of equilibrium distributions can be characterized in terms of equilibrium distributions of the purely local systems. ${ }^{6}$

We recall that a Markov processes is called ergodic if it has a unique invariant distribution and time averages converge to their expected values under the invariant measure.

Theorem 3.4 If the Markov processes with generators $A_{\mu}$ are ergodic with invariant distribution $\nu_{\mu}$, then the following holds:

(i) Every ergodic equilibrium distribution $\mu$ satisfies the fixed point condition $\mu=\nu_{\mu}$.

(ii) The weak limits $\mu$ and $\bar{\mu}$ are equilibria. These equilibria are extremal in the sense that

$$
\underline{\mu}=\inf \left\{\mu: \mu=\nu_{\mu}\right\} \in \mathcal{M}_{0} \quad \text { and } \quad \bar{\mu}=\sup \left\{\mu: \mu=\nu_{\mu}\right\} \in \mathcal{M}_{0} .
$$

(iii) The system has a unique equilibrium if $\mu=\bar{\mu}$, i.e., if the fixed point condition

$$
\mu^{*}=\nu_{\mu^{*}}
$$

has a unique solution:. In this case the equilibrium is globally stable: the macroscopic process converges to $\mu^{*}$ independently of the initial distribution.

Our existence and uniqueness result of equilibrium is applicable whenever the monotone flip rates $c(\eta, a, \mu)$ are strictly positive. In this case a fundamental theorem of statistical mechanics states that the rates can be represented as exponentials of some potential function and that the set of stationary distributions of the Markov process associated with the generator $A_{\mu}$ is given by a set of Gibbs measures; see Blume (1993) and references therein

\footnotetext{
${ }^{6}$ The set $\mathcal{M}_{0}$ is not closed in the weak topology. Hence the weak limit of a sequence of ergodic measures is not necessarily ergodic. Hence the candidate minimal and maximal equilibria, $\underline{\mu}$ and $\bar{\mu}$, are not necessarily ergodic. To guarantee ergodicity we impose an ergodicity condition on the purely local systems.
} 
for details ${ }^{7}$. In particular the Markov process with generator $A_{\mu}$ is ergodic with unique stationary distribution $\nu_{\mu}$ if this set contains a single element. This is guaranteed if Dobrushin's uniqueness condition (Georgii, 1988, Chapter 8) holds, i.e., if the interaction between different agents is sufficiently weak. Under a slightly stronger condition the map $\mu \mapsto \nu_{\mu}$ is a contraction with respect to the metric $d_{r}$ introduced in (5); see Horst (2002) for details. In this case the fixed point condition (14) has a unique solution so that the macroscopic process is globally stable. We state this result in an informal manner and then illustrate it by means of a specific example.

Proposition 3.5 If the interaction between different agents is weak enough, then there exists a unique globally stable equilibrium.

Example 3.6 Consider again the flip rates of Example 2.4. Since $\nu \geq \mu$ implies $m(\nu) \geq$ $m(\mu)$ the monotonicity condition (12) holds because the constants $J_{1}$ and $J_{2}$ are non-negative. Hence when started in the "all +1 " or "all -1 " configuration the macroscopic process settles down in the long run. Furthermore, it is known (Liggett (2005); p. 201) that the Markov process with generator $A_{\mu}$ has a unique stationary distribution $\nu_{\mu}$ if either $\beta$ is sufficiently small or $|h|>J_{1}$. It has been shown by Horst (2002) that the contraction condition

$$
d_{r}\left(\nu_{\mu_{1}}, \nu_{\mu_{2}}\right)<\theta d_{r}\left(\mu_{1}, \mu_{2}\right) \text { for some } \theta<1
$$

holds for some $r>0$ if the Lipschitz constant $L$ in equation (8) is less than one half, i.e., if $\beta \cdot J_{1}$ is sufficiently small. In this case the fixed point condition (14) has a unique solution.

In our model maximal and minimal equilibria exists and these equilibria are identical if the interaction is sufficiently weak. However, we cannot use stochastic approximation algorithms to identify their basins of attractions. Such an approach typically requires some form of limit taking - either by letting the number of agents tend to infinity (Benaim and Weibull 2003) or by letting the "noise" in the agents' choices tend to zero. Foster and Young (1990), Kandori, Mailath and Rob (1993) and Young (1993), for instance, studied the long run dynamics in repeated interaction games with uniform matching where the agents play perturbed best response strategies. For the special case of a $2 \times 2$ coordination game these authored showed that the agents eventually coordinate on the risk-dominant equilibrium when the noise in the choice dynamics tends to zero. Ellison (2000) provides a unified framework for analyzing evolutionary games in discrete time that is flexible enough to allow for both local and global interactions. He shows that the selection of the risk dominant equilibrium "is robust to local vs. global interaction" (p.27). However, his analysis is restricted to finite populations, and

\footnotetext{
${ }^{7}$ Notice that the model of Kosfeld (2002) is not covered by this method. He works with the voter model. In this case the flip rates are not strictly positive and the link to Gibbs distribution theory does not apply.
} 
we argued above that - at least from a technical point of view - all interactions in finite populations are local.

\section{Population Games with Random Matching}

In this section we show that in a model with an infinity of agents the selection of equilibria is not always robust to "local vs. global interaction". Specifically, we introduce an "anonymous" component into the model of Blume $(1993,1995)$ and study its impact on the long run behavior of aggregate play. In Blume's model an agent $a \in \mathbb{A}$ configured in the state $\eta_{t}^{a}$ receives an instantaneous payoff $G\left(\eta_{t}^{a}, \eta_{t}^{b}\right)$ from each of her neighbors $b \in N(a)$. The agent switches his state at Poisson random times where the rate depends on the difference of the payoffs that the different states achieve. For coordination games with payoff functions

$$
G=\left(\begin{array}{cc}
1 & 0 \\
0 & x
\end{array}\right) \quad(0<x<1)
$$

he shows that in the limit of a best response dynamics the agents eventually coordinate on the Pareto optimal equilibrium. Here player $a$ is the row player and $\eta^{a}=-1$ corresponds to choosing the top row. Each agent $b \in N(a)$ is column player and $\eta^{b}=-1$ means she choose the left column.

\subsection{Introducing an anonymous component}

Let us assume that the agents are located on the two-dimensional integer lattice and consider the $2 \times 2$ coordination game with payoff matrix (15). The agents receive a payoff from their nearest neighbors while, at the same time, being matched with four (for reasons of symmetry) other agents whose actions are unobservable. The payoffs from the neighbors and random matches are weighted by a factor $J_{1}$ and $J_{2}$, respectively. The case where $J_{2}=0$ corresponds to a purely local interaction while $J_{1}=0$ yields an interaction of mean-field type.

Given the prevailing average choice $m_{t}$, the action $\eta_{t}^{a}$ at time $t$ yields a payoff $J_{1} G\left(\eta_{t}^{a}, \eta_{t}^{b}\right)$ from each neighbor $b \in N(a)$ and an expected payoff $J_{2}\left\{\frac{1-m_{t}}{2} G\left(\eta_{t}^{a},-1\right)+\frac{1+m_{t}}{2} G\left(\eta_{t}^{a},+1\right)\right\}$ from each agent with whom the agent $a \in \mathbb{A}$ is matched. The overall payoff is thus equivalent to that of a local interaction game with payoff matrix

$$
G_{t}=\left(\begin{array}{cc}
J_{1}+J_{2} \frac{1-m_{t}}{2} & J_{2} \frac{1-m_{t}}{2} \\
x \cdot J_{2} \frac{1+m_{t}}{2} & x \cdot\left(J_{1}+J_{2} \frac{1+m_{t}}{2}\right)
\end{array}\right)
$$

at time $t$. Assuming the same log-linear strategy adjustment process as in Blume (1993) the dynamics of the choices can be described by a system of social interactions with flip rates

$$
c(\eta, a, \mu)=\frac{1}{1+\exp \left[2 \beta \eta^{a}\left(-A(m(\mu))+\frac{B}{4} \sum_{b \in N(a)} \eta^{b}\right)\right]} .
$$


Here $m(\mu)$ denotes the expected action of an individual agent under $\mu$ as defined in (6) and

$$
A(m(\mu))=J_{1}(1-x)+J_{2}\{1-m(\mu)-x(1+m(\mu))\} \quad \text { and } \quad B=J_{1}(1+x) .
$$

As usual, the agents play a best response against the current state of the system when the strength of interactions tends to infinity $(\beta \rightarrow \infty)$ while they chose both actions with equal probability when $\beta=0$.

Remark 4.1 Notice that the feedback from the global interaction affects only the intrinsic value associated with an agent's choice, but has no effect on the payoffs from the neighbors.

In view Theorem 3.4 and Example 3.6 we have the following result.

Proposition 4.2 When started in an "all -1 " or "all +1 " configuration the macroscopic processes settle down in the long run. They converge to the same limit if the interaction between players is sufficiently weak. In this case, the unique equilibrium is globally stable.

\subsection{Breakdown of ergodicity and coordination failure: a numerical analysis}

Unfortunately, it is not possible to solve our measure valued equation for the dynamics of aggregates in closed form. Instead we provide some numerical simulations that suggest a breakdown of ergodicity in the presence of strong interactions and that, due to strong global feedbacks the agents may coordinate on the Pareto inferior equilibrium. Both features are commonly observed in mean-field models; see Hommes (2006) and references therein for a detailed discussion. This suggests that when then the focus is on aggregate behavior, model of local and global interactions are conceptually closer to mean-field models.

\subsubsection{Breakdown of ergodicity}

Figure 1 displays the evolution of the proportions $\left(p_{t}\right)$ of agents configured in state " 1 " over a time span of about 10.000 iterations when started in the "all high" and "all low" configuration, respectively. The simulation is based on an array of $75 \times 75$ agents with $J_{1}=J_{2}=1$ and boundary condition " -1 " when $p_{0}=1$ and " +1 " if $p_{0}=0$. For $x=\frac{1}{2}$ and a sufficiently weak interaction $(\beta=0.1)$, the proportion settles down at approximately $57 \%$ independently of the initial condition as illustrated by Figure 1(a). If the interaction grows stronger $(\beta=0.4)$ ergodicity breaks down. In accordance with Theorem 3.4 (ii) the proportions still converge monotonically when started in an "all +1 " or "all -1 " configuration. However, they converge to different limits. If initially all agent are configured at -1 , the long run proportion decreases to about $90 \%$ while it increases to about $12 \%$ when started in the "all +1 " configuration; see Figure 1(b). 


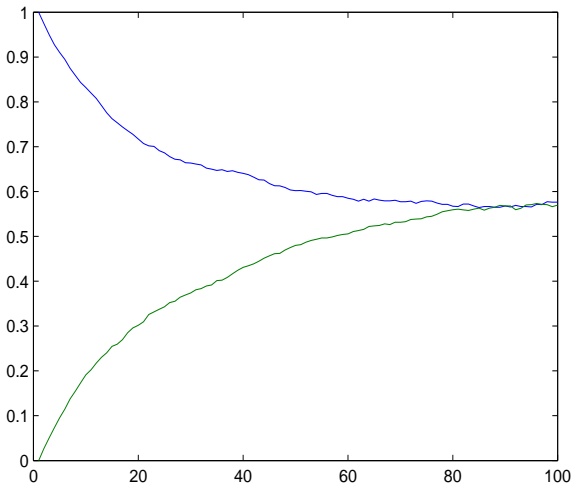

(a) Dynamics for $x=\frac{1}{2}$ and $\beta=0.1$.

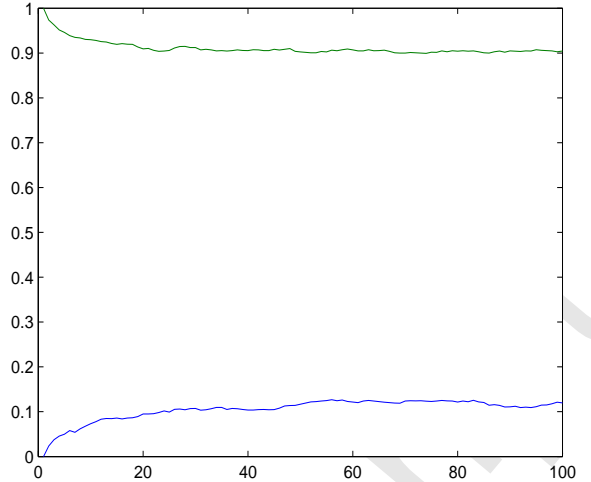

(b) Dynamics for $x=\frac{4}{5}$ and $\beta=0.4$.

Figure 1: Proportion of agents in state " $-1 "$ for $p_{0}=0$ and $p_{0}=1$.

Remark 4.3 We notice that our simulations do by no means imply that for $\beta=0.4$ the dynamics always settles down near the "all +1 " or "all -1 " configuration; there may be other equilibria. In the following section we shall argue, though, that when $\beta$ is large, the system locks into one of two possible steady states, the "all +1 " and "all -1 " equilibrium" and that the final outcome depends strongly on the initial configuration.

\subsubsection{Coordination failure under strong interactions}

Let us now illustrate that in our model the agents may coordinate on the Pareto inferior equilibrium when the interaction is strong (if the interaction is weak there is a unique stable equilibrium). This feature is akin to mean field models. It is not shared by models with only local interactions. In this case the agents always coordinate on the optimal equilibrium: in the benchmark model with frozen macroscopic component $\mu$

$$
\lim _{\beta \rightarrow \infty} \lim _{t \rightarrow \infty} \mathbb{P}\left[\eta_{t}^{a}=-1\right]= \begin{cases}1 & \text { if } A(m(\mu))>0 \\ 0 & \text { if } A(m(\mu))<0\end{cases}
$$

due to Theorem 5.3 in Blume (1993). The fact that the run long behavior of agents in local interaction models depends only on the sign of $A(m(\mu))$ suggests that in a population game with global interactions the agents may eventually fail to coordinate on the Pareto optimal equilibrium. If a large enough proportion of agents is initially configured at " +1 " so that $A\left(m_{0}\right)$ is negative and the interaction is strong an agent configured at "- 1 " has a strong incentive to change their state. This effect may become self-reinforcing: when more agents switch to +1 the external field decreases further thereby increasing an agent's incentive to switch from -1 to +1 . That way, the agents may get trapped in the "all +1 " equilibrium. 


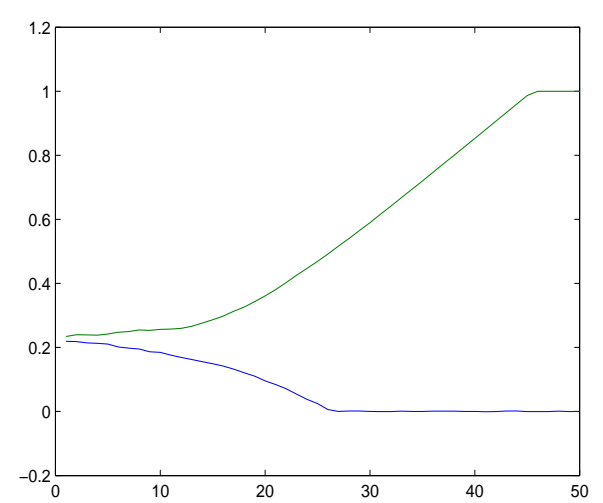

(a) Dynamics for $x=\frac{1}{2} ; p_{0}=.22$ and $p_{0}=0.23$.

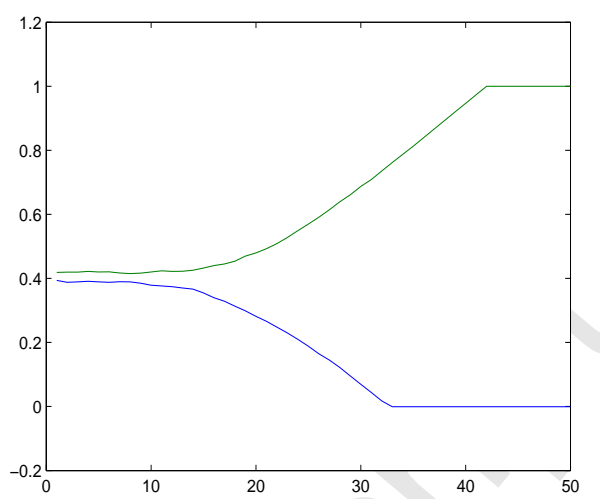

(b) Dynamics for $x=\frac{3}{4} ; p_{0}=.37$ and $p_{0}=.40$.

Figure 2: Proportion of agents in state " $1 " ; \beta=2$; boundary condition "-1".

We leave it for future research to substantiate these arguments in a mathematically rigorous manner and rely instead on a numerical analysis. Figure 2 displays the evolution of the proportion of agents configured in state " 1 " for $\beta=2$ when $x=\frac{1}{2}$ and $x=\frac{3}{4}$, respectively over 5.000 transitions. The simulations show that a slight difference in the initial proportions $p_{0}$ may have a major effect on the long run dynamics: for $x=\frac{1}{2}$ and $p_{0} \approx 23 \%$ the agents coordinate on the unique Pareto optimal equilibrium in the long run while for $p_{0} \approx 22 \%$ the agents eventually settle on the Pareto inferior "all +1 " equilibrium. Furthermore when $x$ increases the "basin of attraction" of the inferior equilibrium grows as "negative" complementarities from global interactions become more powerful. At the same time we see from (17) that there are no negative external effects from global interactions if the impact of the macroscopic component is sufficiently weak. In this case the "all -1" equilibrium will emerge in the long run. Whether or not the agents coordinate on the optimal equilibrium will eventually depend on the relative importance of the global over the local component in the interaction.

\section{Conclusion}

We considered evolutionary models of social interactions with an infinite number of locally and globally interacting agents facing repeated binary choice problems. We showed that the dynamics of aggregate behavior evolves as if it were deterministic. On the level of empirical fields the dynamics macroscopic variables follows a recursive relation. Such a recursion typically cannot be formulated on the level of average actions or empirical distribution of choices. Under the assumption of positive complementarities we established existence and uniqueness 
results of aggregate equilibrium. We showed that the macroscopic process settles down when the system is started in an "all low" or "all high" configuration. While there is generally no reason to assume that the system settles down to a unique limit, the dynamics on the level of aggregate behavior settles down to the same limit if the interaction is weak enough. Our convergence results were applied to a class of local interaction games with random matching. Numerical simulations suggest that when the interaction is sufficiently strong the agents coordinate on an equilibrium of the underlying one-shot game. The qualitative behavior of the dynamics shares many similarities with purely global interactions. Substantiating the numerical results in a mathematically rigorous manner is a purely probabilistic problem well beyond the scope of the present paper and is left for future research. It would also be interesting to prove a central limit theorem to describe the fluctuations of aggregate behavior of large but finite economies around our measure-valued ODE.

\section{A Proofs of the Main Results}

This appendix outlines the construction of the particle process and the proof our existence and uniqueness results of equilibrium. A probability measure on $E$ is called a random field. The space $\mathcal{M}$ of all random fields is compact with respect to the topology of weak convergence and $\mathcal{M}_{h} \subset \mathcal{M}$ is closed. The weak topology is induced by the metric $d_{r}$ in (5) so $\left(\mathcal{M}_{h}, d_{r}\right)$ is a compact metric space; see Horst (2002) for details. The set $\mathcal{L}$ of all local functions $f: E \rightarrow \mathbb{R}$ that depend only on finitely many coordinates is dense in the set of continuous functions with respect to the topology of uniform convergence. Hence a sequence of random fields $\left\{\mu_{t}\right\}$ converges weakly to $\mu \in \mathcal{M}$ if and only if $\lim _{t \rightarrow \infty} \mu_{t}(f)=\mu(f)$ for all $f \in \mathcal{L}$.

\section{A.1 Construction of the microscopic process}

Modifying an approach of Föllmer and Horst (2001) the idea is to define the microscopic process $\left\{\eta_{t}\right\}$ by a process with the empirical field replaced by the solution to some integral equation. To this end, let $\Psi=\left\{\Psi_{t}\right\}$ be a sequence homogeneous random fields on $E$ and consider a time-inhomogeneous process $\left\{\xi_{t}^{\Psi}\right\}$ whose infinitesimal generator at time $t$ is

$$
A_{t} f(\xi) \equiv A_{\Psi_{t}} f(\xi):=\sum_{x} c\left(\xi, x, \Psi_{t}\right)\left[f\left(\xi_{x}\right)-f(\xi)\right] \quad(f \in \mathcal{L})
$$

where the configuration $\xi_{x}$ coincides with $\xi$ at $x \neq u$ and $\xi_{x}^{x}=-\xi^{x}$. The corresponding semigroup is denoted by $\left\{S_{t}^{\Psi}\right\}$. The process describes the evolution of the agents' states when the dynamics of their assessments about the empirical distribution of choices is given by $\Psi$. For any initial distribution $R \in \mathcal{M}_{0}$ it follows from Theorem I.4.15 in Liggett (2005) that the macroscopic process $\left\{R^{\Psi}\left(\xi_{t}^{\Psi}\right)\right\}$ exists almost surely as a sequence of ergodic empirical fields 
and satisfies the integral equation

$$
R_{t}^{\Psi}(f)=R(f)+\int_{0}^{t} R_{u}^{\Psi}\left(A_{\Psi_{u}} f\right) d u \quad \text { and } \quad R_{t} \in \mathcal{M}_{0}
$$

The goal is then to chose an "externality" $\Psi$ that coincides with the sequence of empirical fields generated by the corresponding process of with purely local interactions $\left\{\xi_{t}^{\Psi}\right\}$. This amounts to proving that there exists a unique solution to the integral equation

$$
R_{t}(f)=R(f)+\int_{0}^{t} R_{u}\left(A_{R_{u}} f\right) d u \text { for all } f \in \mathcal{L} .
$$

To this end, one first applies Lemma 1.6.2 in Ethier and Kurtz (1986) to show that the distributions of states depend continuously in the weak topology on the externality. In a second step one uses Assumption 2.3 to prove that for a given starting point $R_{0}$ the equation (18) depends in a Lipschitz continuous (with respect to the metric introduced in (5)) manner on $\Psi$. One can thus prove existence of a unique solution to the integral equation (19) using standard techniques from ordinary differential equations with Lipschitz coefficients. More precisely, we have the following result.

Theorem A.1 Suppose that the flip rates $c(x, \eta, \mu)$ satisfy Assumption 2.3.

(i) There exists a constant $L_{T}$ such that, for any two externalities $\Psi^{1}$ and $\Psi^{2}$ we have

$$
d_{r}\left(S_{t}^{\Psi^{1}}, S_{t}^{\Psi^{2}}\right) \leq L_{T} \int_{0}^{t} d_{r}\left(\Psi_{u}^{1}, \Psi_{u}^{2}\right) d u \quad(t \geq T)
$$

(ii) The integral equation (19) has a unique ergodic solution $\left\{\Psi_{t}^{R}\right\}$ for any $R \in \mathcal{M}_{0}$.

We are now ready to define the particle process with global interaction.

Definition A.2 Let $R$ be an ergodic initial distribution of states. A particle system with macroscopic interaction with initial distribution $R$ is given by the unique Markov process with local interactions associated with the rates $\left\{c\left(\eta, a, \Psi_{t}^{R}\right)\right\}_{\eta \in E, a \in \mathbb{A}}$ and initial distribution $R$.

\section{A.2 Convergence of the Macroscopic Process and Existence of Equilibrium}

In this section we prove a convergence result for attractive spin systems. A spin system is called attractive, if the flip rates $c(\eta, x, \mu)$ satisfy (11). The general theory of attractive spin systems is well developed (Liggett 2005, Chapter III). To state our convergence results let $\mathbf{M}$ be the class of all monotone functions $f$ on $E$ and write $\mu_{1} \leq \mu_{2}$ for two probability measures $\mu_{1}$ and $\mu_{2}$, if $\mu_{1}(f) \leq \mu_{2}(f)$ for all $f \in \mathbf{M}$. The semi-group $S_{t}^{\mu}$ associated with the flip rates $c(\eta, x, \mu)$ maps the class $\mathbf{M}$ into itself, and for any initial distribution $\nu$

$$
\delta_{-1} S_{t}^{\mu} \leq \nu S_{t}^{\mu} \leq \delta_{1} S_{t}^{\mu}
$$


where $\delta_{-1}$ and $\delta_{1}$ denote the Dirac measures putting all mass on the configurations $\eta^{a} \equiv$ -1 and $\eta^{a} \equiv+1$, respectively. Moreover, the weak limits $\underline{\nu}^{\mu}=\lim _{t \rightarrow \infty} \delta_{-1} S_{t}^{\mu}$ and $\bar{\nu}^{\mu}=$ $\lim _{t \rightarrow \infty} \delta_{\mathbf{1}} S_{t}^{\mu}$ exist. Hence, the spin system is ergodic if an only if $\underline{\nu}^{\mu}=\bar{\nu}^{\mu}$ (Liggett 2005, Theorem III.2.3). The semi-groups $\left\{S_{t}^{\mu}\right\}$ and $\left\{S_{t}^{\nu}\right\}$ also satisfy a monotonicity condition with respect to the "frozen" assessments about averages (Liggett (2005), Corollary III.1.7):

$$
\mu_{1} S_{t}^{\mu} \leq \mu_{2} S_{t}^{\nu} \quad \text { for } \quad \mu \leq \nu \quad \text { if } \quad \mu_{1} \leq \mu_{2} .
$$

Proposition A.3 Let $R^{-\mathbf{1}}, R^{\mathbf{1}}$, and $R^{\eta}$ be the macroscopic processes of a monotone system with macroscopic interactions and initial distributions $\delta_{-\mathbf{1}}, \delta_{\mathbf{1}}$ and $\delta_{\eta}$, respectively.

(i) For any $t \geq 0$ and any initial distribution $R \in \mathcal{M}_{0}$ we have $R_{t}^{-\mathbf{1}} \leq R_{t}^{\eta} \leq R_{t}^{\mathbf{1}}$ for $R$-a.e. configuration $\eta \in E_{0}$.

(ii) The processes $\left\{R_{t}^{-1}\right\}$ and $\left\{R_{t}^{\mathbf{1}}\right\}$ are monotone, i.e., for $s \leq t$,

$$
R_{s}^{-1} \leq R_{t}^{-1} \quad \text { and } \quad R_{s}^{1} \geq R_{t}^{1}
$$

(iii) The weak limits $\underline{\mu}:=\lim _{t \rightarrow \infty} R_{t}^{-1}$ and $\bar{\mu}:=\lim _{t \rightarrow \infty} R_{t}^{1}$ exist. In particular,

$$
\lim _{t \rightarrow \infty} R_{t}^{\eta}=\mu \quad \text { if } \quad \underline{\mu}=\bar{\mu}=\mu .
$$

Proof: Let us fix a monotone function $f$ and put $k_{s}^{n}:=\frac{i}{n}$ for $\frac{i}{n} \leq s<\frac{i+1}{n}$. For any $\eta \in \mathbb{E}_{0}$ we define processes $\left\{R_{t}^{n, \eta}\right\}$ of ergodic random fields on $E$ by

$$
R_{t}^{n, \eta}(f):=R(\eta)(f)+\int_{0}^{t} R_{k_{s}^{n}}^{n, \eta}\left(A_{R_{k_{s}^{n}}^{n, \eta} f} f\right) d s
$$

Hence we approximate the macroscopic process by a sequence of macroscopic processes associated with spin systems with local interactions. In fact, on the time interval $\left[\frac{i}{n}, \frac{i+1}{n}\right)$ the systems behaves as if it were purely local. In view of (11) and (12) and the monotonicity properties of locally interacting spin systems theses processes satisfy

$$
R_{s}^{n,-1} \leq R_{t}^{n,-1} \leq R_{t}^{n, \eta} \leq R_{t}^{n, 1} \leq R_{s}^{n, \mathbf{1}}
$$

for all $n \in \mathbb{N}$ and $s \leq t$. A standard argument shows that all the processes $\left\{R_{t}^{n, \eta}\right\}(n \in \mathbb{N})$ are uniformly Lipschitz continuous on compact time intervals. It follows from the theorem of Ascoli and Arzela that they have accumulation points. Continuity of the flip rates shows that each such accumulation point satisfies the integral equation

$$
R_{t}^{\eta}(f)=R(\eta)(f)+\int_{0}^{t} R_{s}^{\eta}\left(A_{R_{s}^{\eta}} f\right) d s
$$


Since this equation has a unique solution, we see that $\lim _{n \rightarrow \infty} R_{t}^{n, \eta}=R_{t}^{\eta}$ for all $t \geq 0$ and the convergence is uniform over compact time intervals. This proves (i) and (ii). In order to establish (iii), let $\mu_{1}$ and $\mu_{2}$ be weak accumulation points of the process $\left\{R_{t}^{-\mathbf{1}}\right\}$ :

$$
\lim _{k \rightarrow \infty} R_{t_{k}}^{-1}=\mu_{1} \quad \text { and } \quad \lim _{k \rightarrow \infty} R_{l_{k}}^{-1}=\mu_{2} .
$$

We may with no loss of generality assume that $\ldots t_{k} \leq l_{k} \leq t_{k+1} \leq l_{k+1} \leq \ldots$. Since the map $t \mapsto R_{t}^{-\mathbf{1}}(f)$ is monotone for any monotone function $f$ we see that

$$
\ldots R_{t_{k}}^{-1}(f) \leq R_{l_{k}}^{-1}(f) \leq R_{t_{k+1}}^{-1}(f) \leq R_{l_{k+1}}^{-1}(f) \leq \ldots
$$

This shows that $\mu_{1}(f)=\mu_{2}(f)$ for all monotone functions $f$, and hence $\mu_{1}=\mu_{2}$. Similar arguments show that the weak $\operatorname{limit}_{\lim } \lim _{t} R_{t}^{1}$ exists.

To state our ergodicity result for attractive particle systems let $\left\{R_{t}\right\}$ be the unique weak solution to the integral equation (19) with initial condition $R_{0}=R$. Since

$$
R_{t+s}(f)=R_{t}(f)+\int_{0}^{s} R_{t+u}\left(A_{R_{t+u}} f\right) d u \quad(f \in \mathcal{L})
$$

weak convergence of the process $\left(R_{t}\right)$ to a random field $R^{*}$ yields

$$
\lim _{t \rightarrow \infty} \int_{0}^{s} R_{t+u}\left(A_{R_{t+u}} f\right) d u=0
$$

for any $f \in \mathcal{L}$, and all $s \in \mathbb{R}_{+}$. Since the flip rates $c(\cdot, \cdot, \mu)$ satisfy Assumption 2.3 we obtain

$$
\lim _{t \rightarrow \infty} \sup _{u}\left\|A_{R_{t+u}} f-A_{R^{*}} f\right\|_{\infty}=0 .
$$

Thus, weak convergence of the macroscopic process yields

$$
\lim _{t \rightarrow \infty} R_{t+u}\left(A_{R_{t+u}} f\right)=R^{*}\left(A_{R^{*}} f\right)
$$

because the functions $A_{R_{t+u}} f$ are uniformly bounded. Hence dominated convergence yields

$$
\begin{aligned}
\lim _{t \rightarrow \infty} \int_{0}^{s} R_{t+u}\left(A_{R_{t+u}} f\right) d u & =\int_{0}^{s} \lim _{t \rightarrow \infty} R_{t+u}\left(A_{R_{t+u}} f\right) d u \\
& =\int_{0}^{s} R^{*}\left(A_{R^{*}} f\right) d u
\end{aligned}
$$

As a result, the limiting random field satisfies the fixed point condition

$$
\mu\left(A_{\mu} f\right)=0 \text { for all } f \in \mathcal{L} .
$$

On the other hand, if $\mu$ is an ergodic equilibrium distribution, then the macroscopic process $\left\{R_{t}\right\}$ is almost surely constant and equal to $\mu$. From the representation (19) of $\left\{R_{t}\right\}$, we thus deduce that $\mu$ satisfies (22). To characterize the class of all limiting distribution and to prove Theorems 3.4 and 3.4 and hence remains to establish the following result. 
Theorem A.4 Suppose that the flip rates satisfy Assumption 2.3. If for any homogeneous random field $\mu$ on $E$ the semi-group $\left\{S_{t}^{\mu}\right\}$ is ergodic with unique stationary distribution $\nu_{\mu}$, then an attractive particle system with global interaction satisfies

$$
\underline{\mu}=\inf \left\{\mu: \mu=\nu_{\mu}\right\} \in \mathcal{M}_{0} \quad \text { and } \quad \bar{\mu}=\sup \left\{\mu: \mu=\nu_{\mu}\right\} \in \mathcal{M}_{0}
$$

In particular, the system is ergodic if there exists a unique $\mu^{*}$ such that

$$
\mu^{*}=\nu_{\mu^{*}}
$$

Proof: The semi-group $\left\{S_{t}^{\mu}\right\}$ is ergodic with unique stationary distribution $\nu_{\mu}$. By Proposition I.2.13 in Liggett (2005) $\nu_{\mu}$ is the only measure that satisfies

$$
\nu_{\mu}\left(A_{\mu} f\right)=0 \text { for all } f \in \mathcal{L} .
$$

Thus, (22) shows that when the process $\left\{R_{t}\right\}$ converges to some random field $\mu^{*}$, the limit must satisfy (24). If the fixed point condition has a unique solution we see that

$$
\underline{\mu}=\bar{\mu}=\mu^{*}
$$

and $\left\{R_{t}\right\}$ converges to $\mu^{*}$, due to Proposition A.3. Moreover, uniqueness of stationary distributions for $\left\{S_{t}^{\mu}\right\}$ implies $\nu_{\mu} \in \mathcal{M}_{0}$ (Liggett (2005), Chapter 2). Thus, for any $\mu$ that satisfies the fixed point condition (24), we that the distribution of the microscopic process does not change through time if the starting point is chosen according to $\mu$. Hence, if $T$ denotes its semi-group, then $\mu T(t)=\mu$. Now, the monotonicity condition yields (23) because

$$
\delta_{-1} T(t) \leq \mu T(t) \leq \delta_{\mathbf{1}} T(t) \text { for all } \quad t \geq 0 .
$$

\section{References}

Allen, F., Gale, D., 2000. Financial contagion. Journal of Political Economy 108, 1-33.

Banerjee, A., 1993. The economics of rumors. Review of Economic Studies 60, 309-327.

Benaim, M., Weibull, J., 2003. Deterministic approximation of stochastic evolution in games. Econometrica 71, 873-903.

Bisin, A., Horst, U. Özgür, O., 2006. Rational expectations equilibria of economies with local interactions. Journal of Economic Theory 127, 74-116. 
Blume, L., 1993. The statistical mechanics of strategic interaction. Games and Economic Behavior 5, 387-424.

Blume, L., 1995. The statistical mechanics of best-response strategy revision. Games and Economic Behavior 11, 111-145.

Brock, W., Durlauf, S., 2001. Discrete choice with social interactions. Review of Economic Studies 68, 235-260.

Durlauf, S., 1993. Non-ergodic economic growth. Review of Economic Studies 60, 349-366.

Durlauf, S., 2000. Basins of attraction, long-run stochastic stability, and the speed of stepby-step evolution. Review of Economic Studies 67, 17-45.

Ethier, S., Kurtz, T., 1986. Markov Processes: Characterization and Convergence. John Wiley, New York.

Föllmer, H., 1974. Random economies with many interacting agents. Journal of Mathematical Economics 1, 51-62.

Föllmer, H., Horst, U., 2001. Convergence of locally and globally interacting Markov chains. Stochastic Processes and Their Applications 96, 99-121.

Foster, D., Young, P., 1990. Stochastic evolutionary games dynamics. Journal of Theoretical Population Biology 38, 219-232.

Georgii, H.O., 1988. Gibbs Measures and Phase Transitions. de Gruyter, Berlin.

Hommes, C., 2006. Heterogeneous agent models in economics and finance. In: Tesfatsion, L., Judd, K. (Eds.). Handbook of Computational Economics, Vol. 2, Amsterdam: NorthHolland, 1109-1186.

Horst, U., 2002. Asymptotics of locally interacting Markov chains with global signals. Advances in Applied Probability 34, 1-25.

Horst, U., 2005. Financial price fluctuations in a stock market model with many interacting agents. Economic Theory 25, 917-932.

Horst, U., 2007. Stochastic Cascades, Credit Contagion, and Large Portfolio Losses. Journal of Economic Behavior and Organization 63, 25-54.

Horst, U. Scheinkman, J., 2006. Equilibria in systems of social interaction. Journal of Economic Theory 130, 44-77. 
Horst, U. Scheinkman, J., 2007. A limit theorem for systems of social interaction. Journal of Mathematical Economics. to appear.

Ioannides, Y., 2006. Topologies of Social Interactions. Economic Theory 28, 559-584.

Ioannides, Y.M. Soetevent, A.R., 2005. Social networking and individual outcomes beyond the mean-field case. Working paper, Tufts University.

Kandori, M., Mailath, G., Rob, R., 1993. Learning, mutation, and long run equilibria in games. Econometrica 61, 29-56.

Kirman, A., 1992. Whom or what does the representative individual represent? Journal of Economic Perspectives 6, 117-136.

Kosfeld, M., 2002. Stochastic strategy adjustment in coordination games. Economic Theory $20,321-339$.

Kosfeld, M., 2005. Rumors and markets. Journal of Mathematical Economics 41, 646-664.

Kurtz, T., 1978. Strong approximation theorems for density dependent Markov chains. Stochastic Processes and Their Applications 6, 223-240.

Liggett, T., 2005. Interacting Particle Systems. Springer-Verlag, Berlin.

Milgrom, R., Roberts, J., 1990. Rationalizability, learning and equilibrium in games with strategic complementarities. Econometrica 58, 1255-1277.

Tanabe, Y., 2006. The propagation of chaos for interacting individuals in a large population. Mathematical Social Sciences 51, 125-152.

Topkis, D., 1979. Equilibrium points in nonzero-sum n-person supermodular games. SIAM Journal on Control and Optimization 17, 773-787.

Topa, G., 2001. Social interaction, local spillover, and unemployment. Review of Economic Studies 68, 261-295.

Vives, X., 1990. Nash equilibrium and strategic complementarities. Journal of Mathematical Economics 19, 305-321.

Young, P., 1993. The evolution of conventions. Econometrica 61, 57-84. 


\title{
Dynamic Systems of Social Interactions
}

\author{
Ulrich Horst* \\ Department of Mathematics \\ Humboldt University Berlin \\ Unter den Linden 6 \\ 10099 Berlin \\ e-mail: horst@math.hu-berlin.de.
}

14th June 2008

\begin{abstract}
We state conditions for existence and uniqueness of equilibria in evolutionary models with an infinity of locally and globally interacting agents. Agents face repeated discrete choice problems. Their utility depends on the actions of some designated neighbors and the average choice throughout the whole population. We show that the dynamics on the level of aggregate behavior can be described by a deterministic measure-valued integral equation. If some form of positive complementarities prevails we establish convergence and ergodicity results for aggregate activities. We apply our convergence results to study a glass of population games with random matching.
\end{abstract}

JEL classification: C63, D50, D71.

Keywords: evolutionary dynamics, social interaction, equilibrium, interacting particle systems, coordination games.

*I would like to thank participants at the 2005 NSF/CEME Mathematical Economics Conference at Berkeley, the SAMSI workshop on Credit Risk, and seminar participants at various institutions for valuable comments. Special thanks are due to Kate Markhvida for assistance with the numerical simulations. Financial support through an NSERC individual research grant is gratefully acknowledged. 